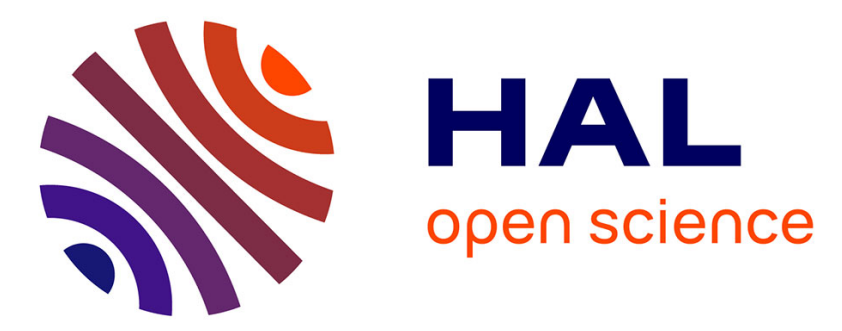

\title{
Élaboration et caractérisation chimique, topographique, tribologique et électrique de films de polyacrylonitrile post-traités en vue d'applications en connectique
} Pascal Newton, Frédéric Houzé, Sophie Noël, Lionel Boyer, Pascal Viel, Gérard Lécayon

\section{To cite this version:}

Pascal Newton, Frédéric Houzé, Sophie Noël, Lionel Boyer, Pascal Viel, et al.. Élaboration et caractérisation chimique, topographique, tribologique et électrique de films de polyacrylonitrile posttraités en vue d'applications en connectique. Journal de Physique III, 1995, 5 (6), pp.661-675. 10.1051/jp3:1995153 . jpa-00249338

\section{HAL Id: jpa-00249338 https://hal.science/jpa-00249338}

Submitted on 1 Jan 1995

HAL is a multi-disciplinary open access archive for the deposit and dissemination of scientific research documents, whether they are published or not. The documents may come from teaching and research institutions in France or abroad, or from public or private research centers.
L'archive ouverte pluridisciplinaire HAL, est destinée au dépôt et à la diffusion de documents scientifiques de niveau recherche, publiés ou non, émanant des établissements d'enseignement et de recherche français ou étrangers, des laboratoires publics ou privés. 
Classification

Physics Abstracts

$68.20-73.60-81.40 \mathrm{P}$

\title{
Élaboration et caractérisation chimique, topographique, tribolo- gique et électrique de films de polyacrylonitrile post-traités en vue d'applications en connectique
}

\author{
Pascal Newton $\left({ }^{1}\right)$, Frédéric Houzé $\left({ }^{1}\right)$, Sophie Noël $\left({ }^{1}\right)$, Lionel Boyer $\left({ }^{1}\right)$, Pascal Viel $\left({ }^{2}\right)$ \\ et Gérard Lécayon $\left({ }^{2}\right)$ \\ ( ${ }^{1}$ Laboratoire de Génie Électrique de Paris, URA CNRS 0127, Groupe Interfaces des Contacts \\ Électriques, Supélec, Plateau de Moulon, 91192 Gif-sur-Yvette Cedex, France \\ $\left({ }^{2}\right)$ DSM-DRECAM-SRSIM, Centre d'Études Nucléaires de Saclay, 91191 Gif-sur-Yvette Cedex, \\ France
}

(Reçu le 19 juillet 1994, révisé le 28 novembre 1994, accepté le 16 décembre 1994)

Résumé. - Un travail interdisciplinaire est mené depuis plusieurs années afin d'élaborer des films fins de polymère traités thermiquement qui remplaceraient la couche métallique terminale classique des connecteurs électriques bas-niveau. Les auteurs montrent dans cet article que des couches de quelques centaines d'Angströms de PolyAcryloNitrile (PAN) greffées sur du nickel galvanique et cuites rapidement dans un four à lampes permettent d'obtenir des revêtements organiques qui possèdent des propriétés électriques et tribologiques intéressantes pour les applications envisagées. Ils présentent les résultats d'investigations, réalisées au niveau moléculaire (caractérisation physico-chimique des films par spectroscopie infra-rouge en incidence rasante IRRAS et spectroscopie de photoélectrons X XPS), au niveau microscopique (microscopie à force atomique AFM), et au niveau macroscopique (mesure de la résistance de contact statique et de la résistance à l'usure), concernant l'évolution chimique et physique de films de PAN ayant subi différents traitements thermiques. Les résultats obtenus pour certains échantillons ont montré des performances très prometteuses en vue d'applications en connectique.

\begin{abstract}
Interdisciplinary work has been carried out for a few years to elaborate thin heat-treated films of polymer in order to replace the usual terminal metallic coatings of low level electrical connectors. The autors show that PolyAcryloNitrile layers a few hundred Angströms thick grafted on electroplated nickel and rapidly heated in a lamp furnace lead to organic coatings which can have adequate electrical and tribological properties for the aimed applications. The authors present in this paper the results of investigations carried out at a molecular scale (chemical characterization of the layers by Infra-Red Reflexion/Absorption Spectroscopy, IRRAS, and $\mathrm{X}$ Photoelectrons Spectroscopy, XPS), at a microscopic scale (topographical characterization by Atomic Force Microscopy, AFM), and at a macroscopic scale (contact resistance measurements and wear resistance measurements), concerning the chemical and the physical evolution of heat-treated PAN layers. These results bring out very promising performances for some of the obtained materials.
\end{abstract}




\section{Introduction}

Cette étude entre dans le cadre plus large d'une recherche visant à réaliser un contact de connecteur électrique bas-niveau dont la couche terminale classique de métal ( $\mathrm{Sn}, \mathrm{Au}, .$.$) serait$ remplacée par un polymère. Cette mise en œuvre d'un polymère nécessite de satisfaire à trois conditions essentielles. Tout d'abord, l'utilisation, lors de la phase de recherche, d'un substrat métallique de type industriel. Ensuite, le film organique doit être couvrant et adhérent afin de préserver le substrat métallique de l'usure et de la corrosion. Enfin, le polymère doit conduire à une faible résistance électrique de contact. Face à ces contraintes, le PolyAcryloNitrile (PAN) présente des propriétés qui apparaissent très prometteuses. En effet, le dépôt du PAN par électropolymérisation conduit à la formation d'un film mince greffé solidement au substrat métallique. De plus, le traitement thermique du PAN permet d'améliorer la conductivité électrique des films de plusieurs ordres de grandeur. La cuisson du polymère constitue donc une étape essentielle dans la fabrication de films utilisables en connectique. Les diverses transformations subies par le matériau au cours du traitement thermique ont été suivies à différentes échelles d'observation. L'évolution de la structure moléculaire du polymère a été caractérisée au moyen des spectroscopies IRRAS et XPS. L'observation microscopique de l'extrême surface des films au moyen d'un AFM nous a révélé les transformations physiques qui interviennent lors du traitement. Enfin, des mesures de résistance de contact et de résistance à l'usure des différents échantillons nous ont permis de suivre les modifications des propriétés macroscopiques des échantillons. L'efficacité des différents traitements thermiques en vue d'applications en connectique est ainsi attestée.

\section{Fabrication des échantillons}

2.1. SynthÈse DES FILMS. - Les films de PAN sont déposés par électropolymérisation sous polarisation cathodique. Cette méthode de synthèse originale a été développée au C.E.N. Saclay $[1,2]$. Elle consiste à provoquer une interaction acide/base au sens de Lewis entre la surface métallique rendue nucléophile par polarisation cathodique et l'extrémité électrophile d'un monomère vinylique (l'acrylonitrile). Plusieurs phénomènes interviennent alors : (i) sous polarisation, il se produit l'orientation de la molécule d'acrylonitrile dans le champs de double couche électrochimique. Cette molécule de monomère présente alors son extrémité vinylique vers la surface métallique ; (ii) dans ces conditions, la mise en commun d'un doublet d'électrons de la cathode avec la molécule d'acrylonitrile conduit à la formation d'un anion organique lié chimiquement à la phase minérale ; (iii) l'anion, initiateur d'une réaction de polymérisation anionique classique, amorce ensuite la croissance de chaînes de polymère linéaires greffées sur l'électrode. On obtient par cette technique des films de PAN couvrants et très adhérents puisque de véritables liaisons chimiques existent à l'interface polymère/métal. Cette méthode permet un dépôt homogène et reproductible d'un film fin de PAN sur un substrat métallique qui peut être de forme complexe dans le cadre d'applications industrielles (peignes de connecteurs...).

La méthode détaillée de la synthèse de nos échantillons a déjà été décrite [3]. Les films organiques sont déposés sur des coupons de laiton $(70 \mathrm{~mm} \times 10 \mathrm{~mm} \times 1 \mathrm{~mm}$ ) recouverts de $1,2 \mu \mathrm{m}$ de nickel galvanique. Ce substrat est de même nature que ceux utilisés dans l'industrie avant le dépôt d'une couche terminale d'or. L'épaisseur des films, contrôlée par ellipsométrie, se situe entre 500 et $600 \AA$. Cette épaisseur constitue un compromis entre un film épais qui permet d'atténuer la rugosité du substrat métallique et un film fin qui devrait favoriser le transport électronique et diminuer ainsi la résistance de contact de l'échantillon. 
2.2. Traitement thermique Rapide. - La pyrolyse du PAN est un procédé industriel courant intervenant dans la fabrication de la fibre de carbone [4]. Au cours du traitement thermique, le PAN évolue vers une structure cyclisée et conjuguée, améliorant sa conductivité électrique de plusieurs ordres de grandeur [5]. Ce traitement thermique consiste habituellement en une stabilisation du polymère par un chauffage à l'air avec une température comprise entre 200 et $300^{\circ} \mathrm{C}$, puis en une montée à haute température $\left(\geq 1000^{\circ} \mathrm{C}\right)$ sous vide ou en atmosphère inerte. Les montées en température sont lentes $\left(1 \mathrm{à} 2^{\circ} \mathrm{C} / \mathrm{mn}\right)$ et les cuissons longues (plusieurs heures) $[6,7]$.

Dans le cas de nos travaux, nous avons choisi de réaliser le traitement thermique dans un four à lampes irradiant dans le domaine de l'infra-rouge et du visible. En effet, ce dispositif permet un traitement thermique rapide qui évite la dégradation du substrat métallique de laiton. De plus, cette méthode rapide, qui permet de s'affranchir des lourdes techniques du vide, apparait facilement implantable sur les lignes de production industrielles de connecteurs.

Les cycles de cuisson s'effectuent sous flux d'argon et consistent en une rapide montée en température $\left(200^{\circ} \mathrm{C} / \mathrm{s}\right)$ jusqu'à un palier à $350,400,450$, ou $500^{\circ} \mathrm{C}$ pendant $10 \mathrm{~s}$ ou $400^{\circ} \mathrm{C}$ pendant $30 \mathrm{~s}$. L'ouverture de la porte du four provoque une "trempe" qui ramène les films à la température ordinaire, en moins d'une minute, à l'air.

Des mesures d'épaisseur réalisées par ellipsométrie montrent que la cuisson des films s'accompagne d'une diminution de leur épaisseur. On passe ainsi de $550 \AA$ pour un film cru à $450 \AA$ pour l'échantillon cuit à $400^{\circ} \mathrm{C}$ pendant $10 \mathrm{~s}$, et à $240 \AA$ pour celui cuit à $400^{\circ} \mathrm{C}$ durant $30 \mathrm{~s}$.

\section{Caractérisation physico-chimique des échantillons}

Les films traités thermiquement sont caractérisés par spectroscopie de réflexion/absorption infra-rouge en incidence rasante (IRRAS) et par spectroscopie de photoélectrons X (XPS).

3.1. ANALYSE INFRA-ROUGE. - Les spectres infra-rouge de reflexion/absorption en incidence rasante $\left(5^{\circ}\right)$ sont acquis avec un spectromètre BRÜCKER IFS 66 . Chaque spectre résulte de 256 balayages avec une résolution de $2 \mathrm{~cm}^{-1}$ Le détecteur est de type MCT (Mercure-CadmiumTelluride). L'évolution du polymère observée en IRRAS apparaît sur la figure 1. On constate en premier lieu que le spectre de bandes fines, résultant de la vibration d'atomes de chaînes linéaires isolées de PAN, évolue vers un spectre de bandes plus larges, traduisant les vibrations collectives d'atomes au sein d'un vaste réseau moléculaire. La figure la montre le spectre du film de PAN cru où apparaissent les trois bandes caractéristiques correspondant aux vibrations de déformation $\delta \mathrm{CH}_{2}\left(1455 \mathrm{~cm}^{-1}\right)$ et de d'élongation $\nu_{\mathrm{as}} \mathrm{CH}_{2}\left(2940 \mathrm{~cm}^{-1}\right)$ des groupements méthylènes, et aux vibrations d'élongation $\nu \mathrm{CN}\left(2240 \mathrm{~cm}^{-1}\right)$ des groupements nitriles. La structure correspondante du polymère est représentée schématiquement sur la figure 2a. Le spectre $1 \mathrm{~b}$ montre que le film traité à $350^{\circ} \mathrm{C}$ durant $10 \mathrm{~s}$ se distingue du PAN cru par l'apparition d'une bande à $2190 \mathrm{~cm}^{-1}$ qui révèle une structure énaminonitrile résultant d'un début de réticulation inter-chaîne du polymère [8]. L'échantillon traité à $400^{\circ} \mathrm{C}$ durant $10 \mathrm{~s}$ a subi de plus profondes transformations. Les variations principales de son spectre (Fig. 1c) se révèlent par l'apparition de quatre nouvelles bandes à 1250,1385, 1600 et $1640 \mathrm{~cm}^{-1}$ qui correspondent aux vibrations de valence des doubles liaisons vinyliques et imines conjuguées au sein d'une structure de cycles carbonés. La figure $2 b$ illustre cette évolution résultant de la cyclisation intrachaîne des groupements nitriles. A ce stade, le film de polymère est constitué d'un mélange des structures $2 \mathrm{a}$ et $2 \mathrm{~b}$. Les traitements thermiques plus poussés conduisent à la diminution (Fig. 1d), puis à la complète disparition (Figs. 1e et 1f) de la bande attribuée aux groupements nitriles $\nu \mathrm{CN}\left(2240 \mathrm{~cm}^{-1}\right)$. La forte diminution de l'intensité de la bande $\delta \mathrm{CH}_{2}\left(1455 \mathrm{~cm}^{-1}\right)$ montre que les groupements $\mathrm{CH}_{2}$ ont été consommés dans une réaction de déshydrogénation 


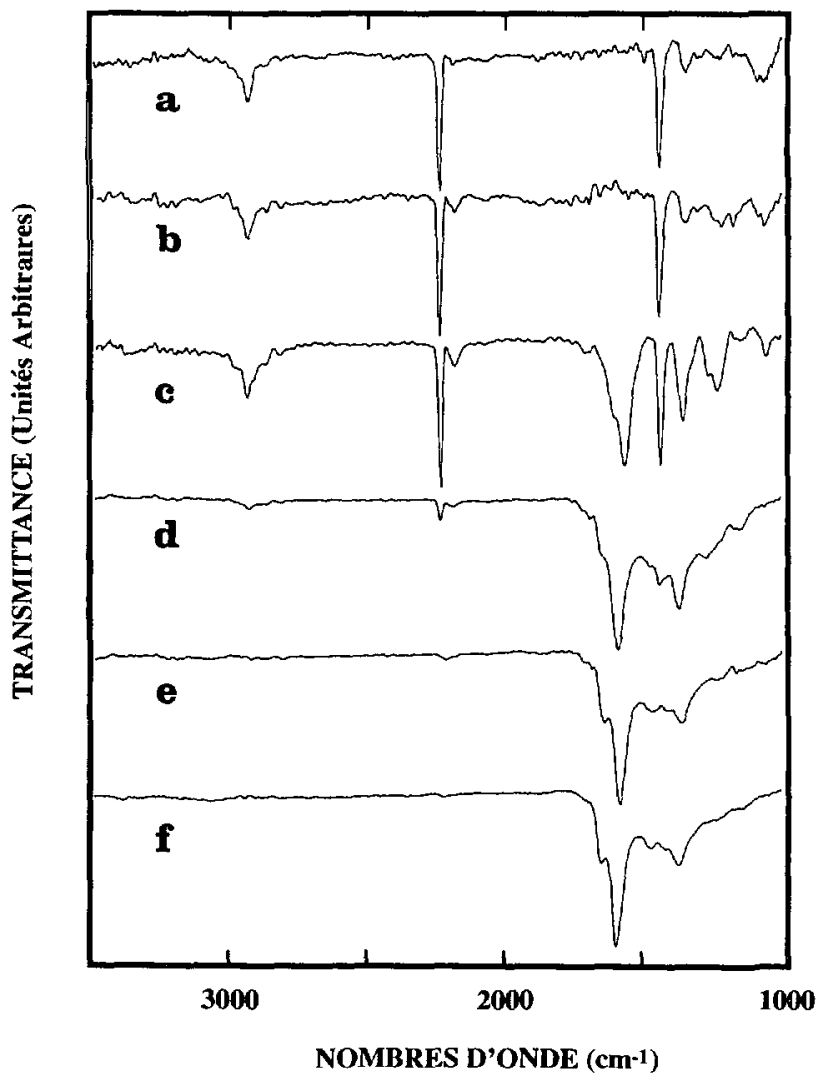

Fig. 1. - Spectres IRRAS des six types d'échantillons étudiés. (a) : PAN cru ; (b) : PAN cuit $350{ }^{\circ} \mathrm{C} / 10 \mathrm{~s}$; (c) : PAN cuit $400{ }^{\circ} \mathrm{C} / 10 \mathrm{~s}$; (d) : PAN cuit $450{ }^{\circ} \mathrm{C} / 10 \mathrm{~s}$; (e) : PAN cuit $500{ }^{\circ} \mathrm{C} / 10 \mathrm{~s}$; (f) : PAN cuit $400^{\circ} \mathrm{C} / 30 \mathrm{~s}$.

[IRRAS spectra of the six studied samples. (a): Raw PAN; (b): PAN heated $350{ }^{\circ} \mathrm{C} / 10 \mathrm{~s}$; (c): PAN heated $400{ }^{\circ} \mathrm{C} / 10 \mathrm{~s}$; (d): PAN heated $450^{\circ} \mathrm{C} / 10 \mathrm{~s}$; (e): PAN heated $500 \mathrm{C} / 10 \mathrm{~s}$; (f): PAN heated $\left.400^{\circ} \mathrm{C} / 30 \mathrm{s.}\right]$

du squelette aliphatique conduisant à la formation de liaisons insaturées $\mathrm{C}=\mathrm{C}$. Le PAN a donc évolué vers le vaste réseau réticulé tridimensionnel de cycles carbonés riches en doubles liaisons conjuguées $(\mathrm{C}=\mathrm{C}$ et $\mathrm{C}=\mathrm{N})$ représenté schématiquement sur la figure $2 \mathrm{~d}[9,10]$.

3.2. ANALySE EN SPECTROSCOPIE DE PHOTOÉlECtrons X. - L'analyse infra-rouge rend compte de tout le volume du polymère. Par une méthode complémentaire d'analyse de surface, l'XPS, nous avons caractérisé les premières couches atomiques des films $(\sim 50 \AA)$. Les mesures ont été réalisées sur un système RIBER MAC 2 en utilisant la raie $K \alpha$ du magnésium et avec une résolution constante de $0,5 \mathrm{eV}$. La figure 3 montre les spectres ainsi obtenus des niveaux électroniques de cœur C1s et N1s. Pour les premiers traitements thermiques (Figs. 3b à 3d), les spectres apparaissent peu différents de ceux du film de PAN cru. En revanche, les traitements thermiques plus poussés (Figs. 3e et 3f) font apparaître des modifications des pics C1s et N1s.

Ainsi, le pic $\mathrm{C} 1 \mathrm{~s}$ de l'échantillon traité à $500^{\circ} \mathrm{C}$ durant $10 \mathrm{~s}$ présente deux composantes différentes. La première, avec une énergie de liaison de $285,4 \mathrm{eV}$ et une largeur à mi- hauteur de $1,6 \mathrm{eV}$, peut être attribuée à des cycles aromatiques comme le confirme un petit "shake-up" 
<smiles>CC(C#N)CC(C#N)CC(C#N)CC(C)C#N</smiles>

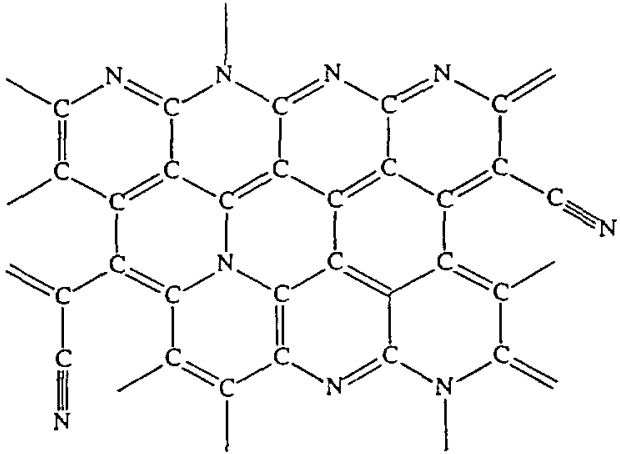

a.<smiles>C=c1cc2cc3cc4cc(C)c(C)nc4nc3nc2nc1=C</smiles>

b

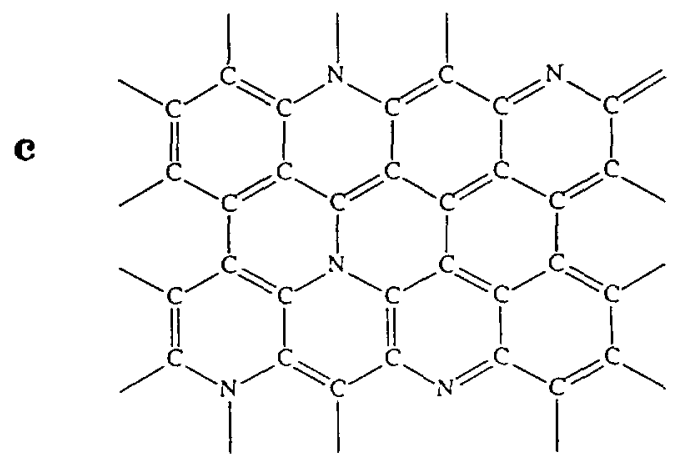

Fig. 2. - Schéma moléculaire illustrant les principales étapes de l'évolution du PAN au cours du recuit.

[Molecular schema illustrating the main steps of the PAN thermal structurization.]

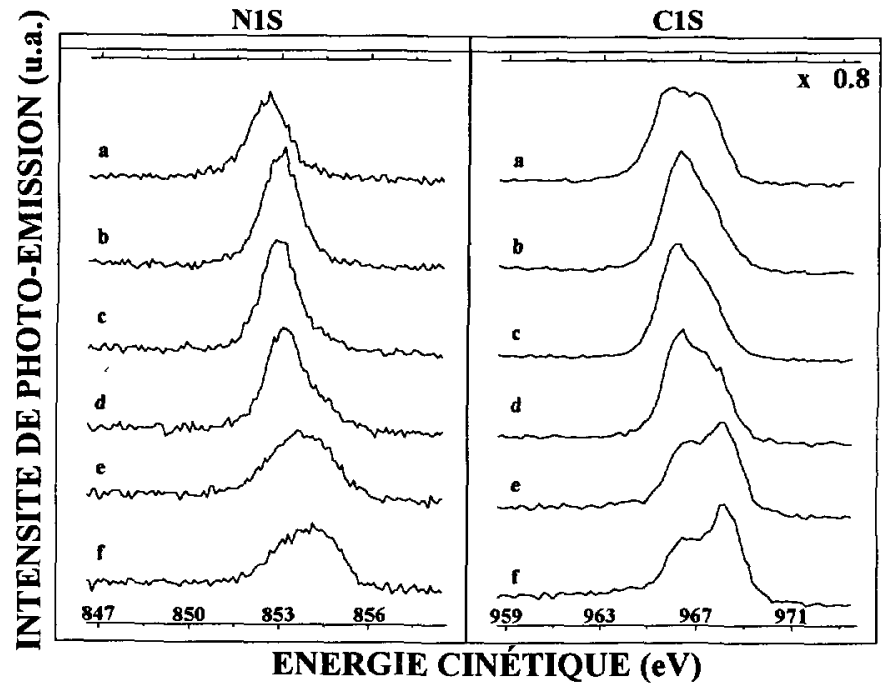

Fig. 3. - Spectres XPS des six types d'échantillons étudiés. (a) : PAN cru ; (b) : PAN cuit $350^{\circ} \mathrm{C} / 10 \mathrm{~s}$; (c) : PAN cuit $400^{\circ} \mathrm{C} / 10 \mathrm{~s} ;$ (d) : PAN cuit $450^{\circ} \mathrm{C} / 10 \mathrm{~s} ;\left(\right.$ e) : PAN cuit $500^{\circ} \mathrm{C} / 10 \mathrm{~s} ;$ (f) : PAN cuit $400^{\circ} \mathrm{C} / 30 \mathrm{~s}$.

[XPS spectra of the six studied samples. (a): Raw PAN; (b): PAN heated $350{ }^{\circ} \mathrm{C} / 10$ s; (c): PAN heated $400{ }^{\circ} \mathrm{C} / 10 \mathrm{~s}$; (d): PAN heated $450{ }^{\circ} \mathrm{C} / 10 \mathrm{~s}$; (e): PAN heated $500{ }^{\circ} \mathrm{C} / 10 \mathrm{~s}$; (f): PAN heated $\left.400^{\circ} \mathrm{C} / 30 \mathrm{~s}.\right]$

à $292,4 \mathrm{eV}$ dû aux transitions $\pi \rightarrow \pi^{*}$. Le second pic, centré à $287,1 \mathrm{eV}$ avec une largeur à mihauteur de $1,7 \mathrm{eV}$, correspond à des atomes de carbone impliqués dans différents groupements azotés. La largeur à mi-hauteur du pic N1s passe de $1,6 \mathrm{eV}$ pour l'échantillon traité à $400^{\circ} \mathrm{C}$ pendant $10 \mathrm{~s}$, à $2,5 \mathrm{eV}$ pour l'échantillon traité à $400^{\circ} \mathrm{C}$ pendant $30 \mathrm{~s}$. Ce pic résulte du 

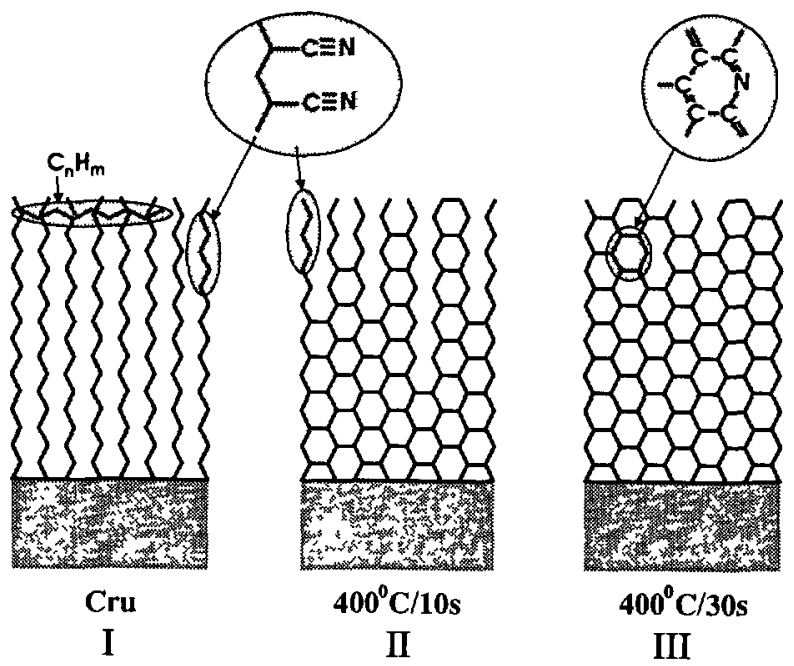

Fig. 4. - Représentation schématique montrant l'hétérogénéité de l'évolution de la structure moléculaire des films de PAN au cours d'un traitement thermique rapide.

[Schematic view of the molecular evolution of heat-treated PAN films.]

chevauchement de plusieurs pics difficilement discernables d'atomes d'azote impliqués dans différentes fonctions chimiques. Quantitativement, les rapports des concentrations atomiques de carbone et d'azote, corrigées par les facteurs de sensibilité, ont été calculés pour chaque échantillon. Ils révèlent que la quantité d'azote contenue dans la couche superficielle analysée a diminué de $10 \%$ après un traitement thermique de $500{ }^{\circ} \mathrm{C} / 10 \mathrm{~s}$ et de $25 \%$ après $400{ }^{\circ} \mathrm{C} / 30 \mathrm{~s}$. Ces analyses montrent que la structure des films traités est un mélange de cycles carbonés plus ou moins azotés selon l'intensité du traitement thermique subi.

Ces résultats confirment l'évolution chimique du polymère montrée par l'analyse Infra-Rouge. Cependant, la modification des spectres XPS intervient à des températures plus hautes que celle des spectres infra-rouge. La comparaison des résultats de chaque méthode révèle l'hétérogénéité de la transformation thermique des films, qui est plus avancée en profondeur qu'en surface, et met en évidence l'existence d'un gradient de température dans l'épaisseur des films lors du traitement thermique. La "trempe" subie par les échantillons en fin de traitement peut expliquer ce phénomène. En effet, lors de l'ouverture de la porte du four, l'air refroidit par convection la surface du film, tandis que le substrat métallique continue de chauffer par conduction le polymère d'interface. Au regard des résultats obtenus par analyses en IRRAS et XPS de nos films, nous proposons sur la figure 4 une représentation schématique de leur évolution au cours du traitement thermique.

\section{Caractérisation topographique}

L'étude de la surface de nos échantillons au moyen d'un microscope à force atomique (AFM) nous permet de caractériser la topographie des films et de savoir si l'évolution chimique de ceux-ci s'accompagne d'une modification notable du relief de la surface.

Nous avons étudié par cette technique trois échantillons de polymère ayant subi différents traitements thermiques et un coupon du substrat de nickel obtenu par dissolution du polymère 

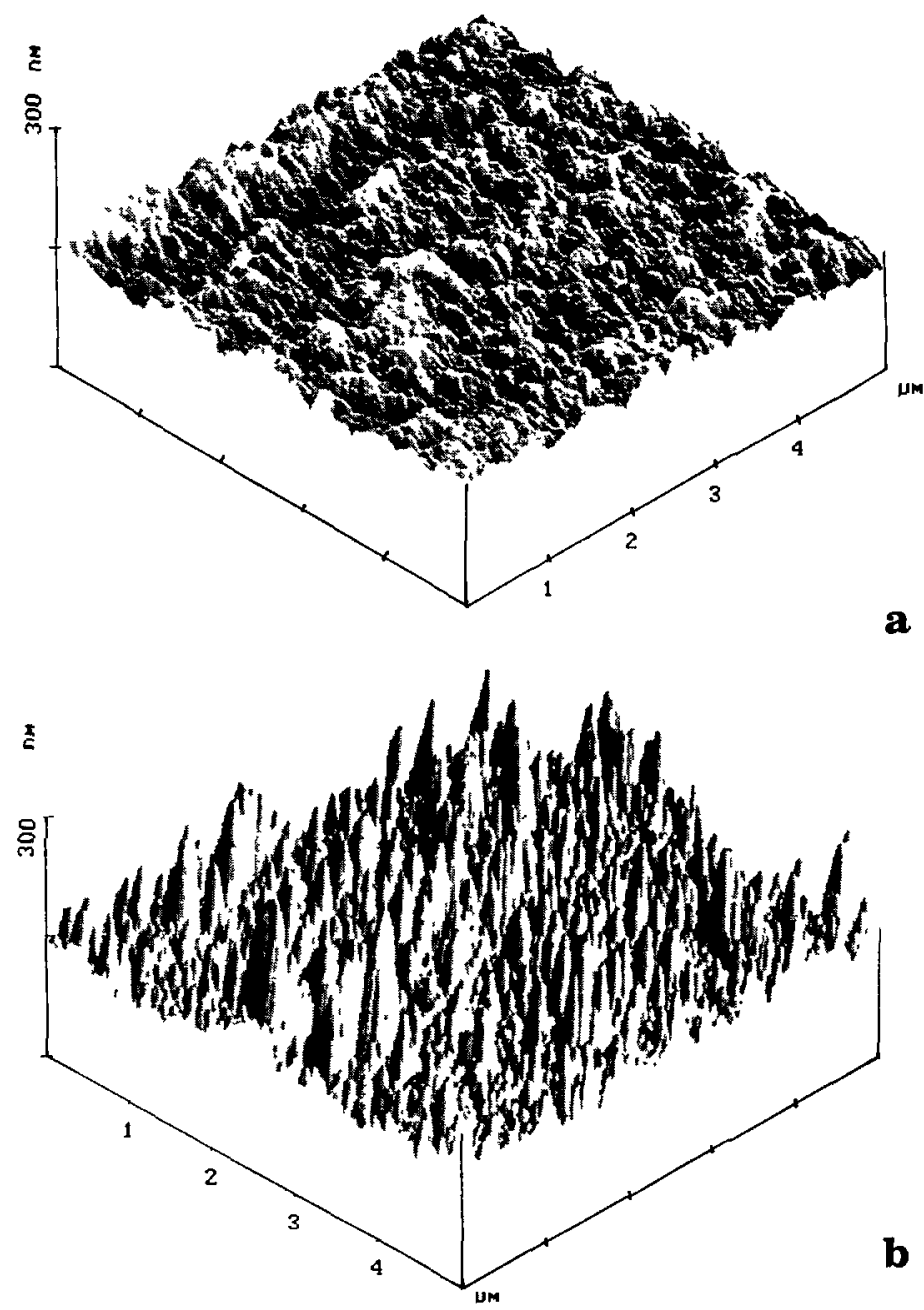

Fig. 5. - Aspect topographique du substrat de nickel et de trois types de films, observés par microscopie à force atomique. (a) : Substrat de nickel ; (b) : PAN cru ; (c) : PAN cuit $400{ }^{\circ} \mathrm{C} / 10 \mathrm{~s}$; (d) : PAN cuit $400{ }^{\circ} \mathrm{C} / 30 \mathrm{~s}$.

[Topographical aspect of the nickel substrate and of three different types of films observed by Atomic Force Microscopy. (a): Nickel substrate; (b): Raw PAN; (c): PAN heated $400^{\circ} \mathrm{C} / 10 \mathrm{~s}$; (d): PAN heated $400{ }^{\circ} \mathrm{C} / 30 \mathrm{~s}$.]

dans la diméthylformamide. Sur chaque type de coupon ont été explorées 10 zones de $5 \times 5 \mu \mathrm{m}$ choisies au hasard. L'aspect des images obtenues a été à chaque fois reproductible et cohérent. Nous avons calculé pour chaque zone les principaux paramètres de rugosité afin d'obtenir des données quantitatives sur le relief de chaque type de coupon. Les valeurs moyennes les plus significatives sont rassemblées dans le tableau I.

La figure 5 a représente la surface de nickel. Nous avons contrôlé par XPS que le film de PAN a bien été totalement dissous dans le solvant. Les figures $5(b, c, d)$ montrent respectivement les surfaces d'un film cru et de films cuits à $400^{\circ} \mathrm{C}$ pendant 10 et $30 \mathrm{~s}$. Il faut souligner que la différence entre les échelles horizontale $(1 \mu \mathrm{m} /$ division) et verticale $(150 \mathrm{~nm} /$ division $)$ exalte 

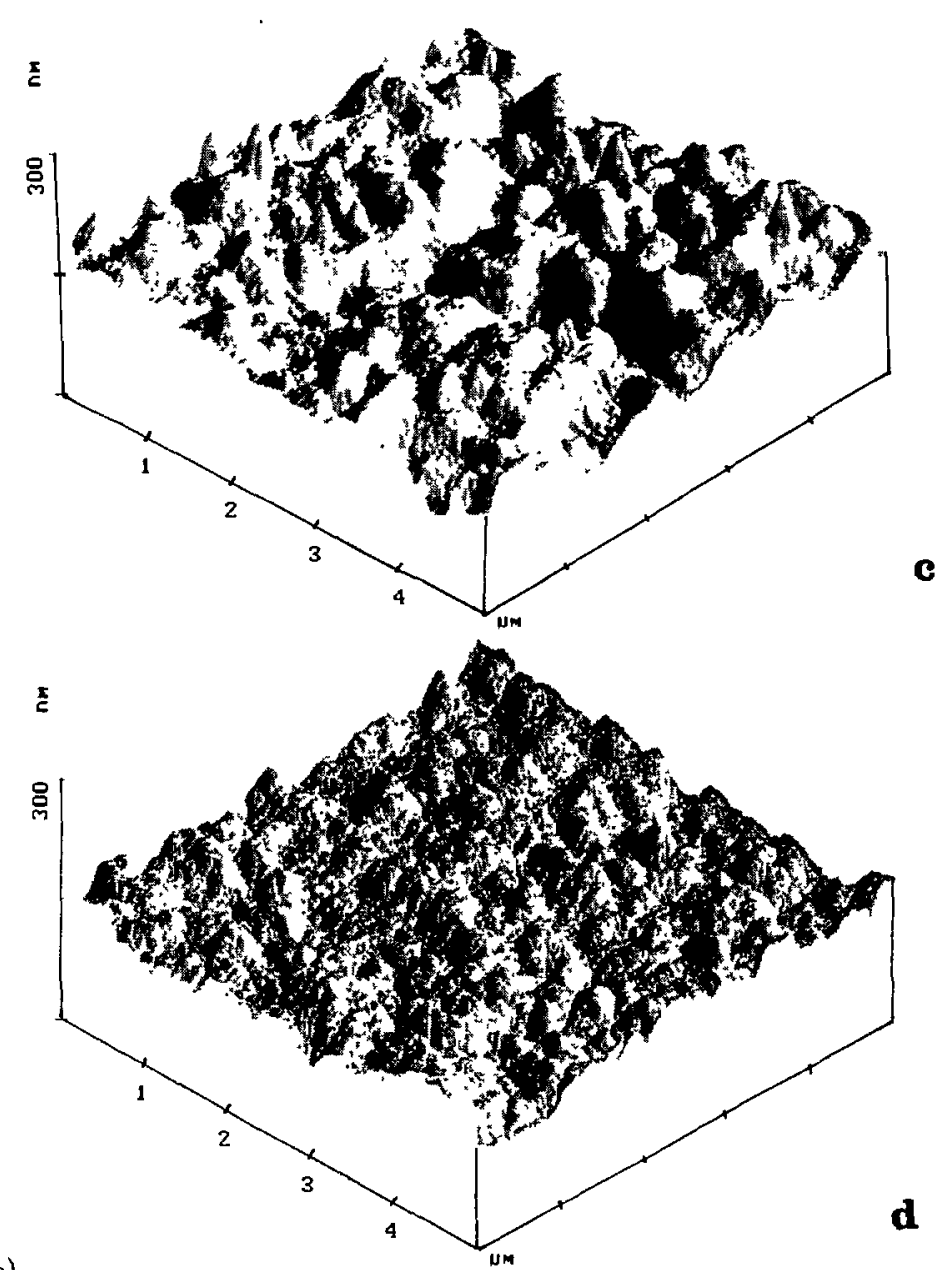

Fig. 5. - (suite)

d

Tableau I. - Valeurs moyennes, sur 10 images $5 \mu m \times 5 \mu m$, des paramètres de rugosité les plus significatifs. $R_{\mathrm{a}}=$ rugosité arithmétique moyenne, $R_{\mathrm{q}}=$ rugosité quadratique moyenne, Nb pics $>250 \AA=$ nombre de pics plus hauts que $250 \AA$ par rapport au plan moyen de l'image, Hmoy pics $>250 \AA=$ moyenne des hauteurs des pics plus hauts que $250 \AA$ par rapport au plan de l'image.

[Mean values, calculated on ten $5 \mu \mathrm{m} \times 5 \mu \mathrm{m}$ pictures, of the most significant roughness parameters. $R_{\mathrm{a}}=$ mean roughness, $R_{\mathrm{q}}=$ standard deviation, Nb pics $>250 \AA=$ number of peaks higher than $250 \AA$ as compared to the mean plane of the picture, Hmoy pics $>250 \AA=$ mean height of the peaks higher than $250 \AA$ as compared to the mean plane of the picture.]

\begin{tabular}{|c|c|c|c|c|}
\cline { 2 - 5 } \multicolumn{1}{c|}{} & $\mathrm{Ra}(\AA)$ & $\mathrm{Rq}(\AA)$ & Nb pics $>250 \AA$ & Hmoy pics $>250 \AA$ \\
\hline Nickel & 94 & 119 & 43 & $289 \AA$ \\
\hline PAN cru & 167 & 224 & 252 & $427 \AA$ \\
\hline Film $400^{\circ} \mathrm{C} / 10 \mathrm{~s}$ & 98 & 126 & 30 & $355 \AA$ \\
\hline Film $400^{\circ} \mathrm{C} / 30 \mathrm{~s}$ & 122 & 155 & 24 & $307 \AA$ \\
\hline
\end{tabular}


la pente des aspérités des surfaces observées. Le relief apparaît ainsi environ 7 fois plus haut qu'il ne l'est en réalité.

La figure $5 \mathrm{~b}$ montre que la surface du polymère cru apparaît très rugueuse. La topographie du PAN semble se décomposer en deux niveaux distincts. On observe de nombreuses aspérités réparties sur l'ensemble de la surface dont la hauteur, très variable, peut atteindre $2000 \AA$. Entre ces hautes aspérités apparaît un second niveau topographique constitué de petites aspérités dont la hauteur ne dépasse pas quelques dizaines de nanomètres. Toutes ces irrégularités de surface sont exclusivement constituées de PAN comme l'atteste l'analyse XPS de la surface : le polymère est parfaitement couvrant.

Les figures 5 (c et $\mathrm{d}$ ) montrent la diminution régulière du nombre et de la hauteur moyenne des aspérités avec le traitement thermique, en même temps que le caractère de plus en plus trapu du relief restant. Les données du tableau I confirment et précisent cette analyse qualitative. Il y a en moyenne sur la surface du film le plus cuit 10 fois moins de pics d'altitude supérieure à $250 \AA$ que sur le PAN cru , la hauteur de ces pics étant environ plus faible de $30 \%$. L'évolution des paramètres standard $R_{\mathrm{a}}$ (rugosité arithmétique moyenne) et $R_{\mathrm{q}}$ (rugosité quadratique moyenne) en fonction du degré de cuisson traduit quant à elle l'aspect de plus en plus massif des pics subsistants.

La diminution d'épaisseur des films, évoquée au paragraphe 2, confirme l'évolution vers une structure plus dense et plus uniforme que celle du polymère cru.

Au cours d'un recuit rapide, tel que nous le pratiquons, la structure moléculaire du film de PAN subit une réticulation et évolue vers une forme de type fibre de carbone ou pyrolitique. Ces modifications se font en un temps extrêmement court et hors équilibre. Il est donc très délicat d'évoquer les paramètres thermodynamiques habituels tels que point de fusion et point de transition vitreuse. On peut citer à ce sujet Krigbaum et al. [10] qui déterminent un point de fusion à $317^{\circ} \mathrm{C}$ et une température de transition vitreuse à $100^{\circ} \mathrm{C}$, en extrapolant des mesures de dilatométrie réalisées sur des solutions diluées de PAN. Mentionnons encore Dunn et al. [11] qui ont mesuré des points de fusion de PAN pur par analyse thermique différentielle en chauffant leurs échantillons rapidement $\left(40^{\circ} \mathrm{C} / \mathrm{mn}\right)$. Ils atteignent ainsi la fonte du polymère à $316{ }^{\circ} \mathrm{C}$ avant que sa dégradation n'intervienne. Nous sommes, avec notre technique de recuit rapide et de trempe, dans des conditions favorables à une transformation physique (ramollissement et étalement) du film préalable à sa transformation chimique. On peut donc supposer raisonnablement qu'un film chauffé à $200^{\circ} \mathrm{C} / \mathrm{s}$ jusqu'à $400^{\circ} \mathrm{C}$ puis maintenu à cette température pendant 10 ou $30 \mathrm{~s}$, s'est étalé sur la surface avant d'être transformé chimiquement.

\section{Propriétés électriques et tribologiques des films}

Nous avons choisi, afin de caractériser les propriétés électriques et tribologiques de nos échantillons, de simuler la géométrie complexe d'un contact de connecteur électrique par un contact idéalisé sphère/plan de même dimension (Fig. 6). La force d'appui de la sphère sur le plan est du même ordre de grandeur que celle qui s'exerce entre les deux membres du contact de connecteur.

5.1. Mesures DE RÉSISTANCE DE CONTACT. - Le dispositif expérimental utilisé a été mis au point au LGEP et est représenté schématiquement sur la figure 7 . Nos échantillons, décrits précédemment, constituent le plan de contact. Les sphères sont des billes de cuivre-béryllium de $3,2 \mathrm{~mm}$ de diamètre recouvertes de $5 \mu \mathrm{m}$ de nickel électrolytique puis de $2 \mu \mathrm{m}$ d'or-cobalt. Le montage électrique réalisé est du type "quatre fils", ce qui permet de mesurer la tension près du contact. Les essais sont réalisés pour un contact statique, évitant tout cisaillement du film, 


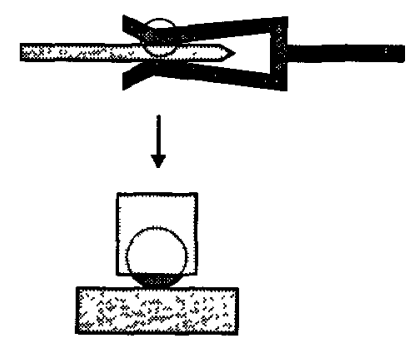

Fig. 6. - Modèle sphère/plan d'un contact électrique.

[Ball on plane simulation of a real electrical contact.]

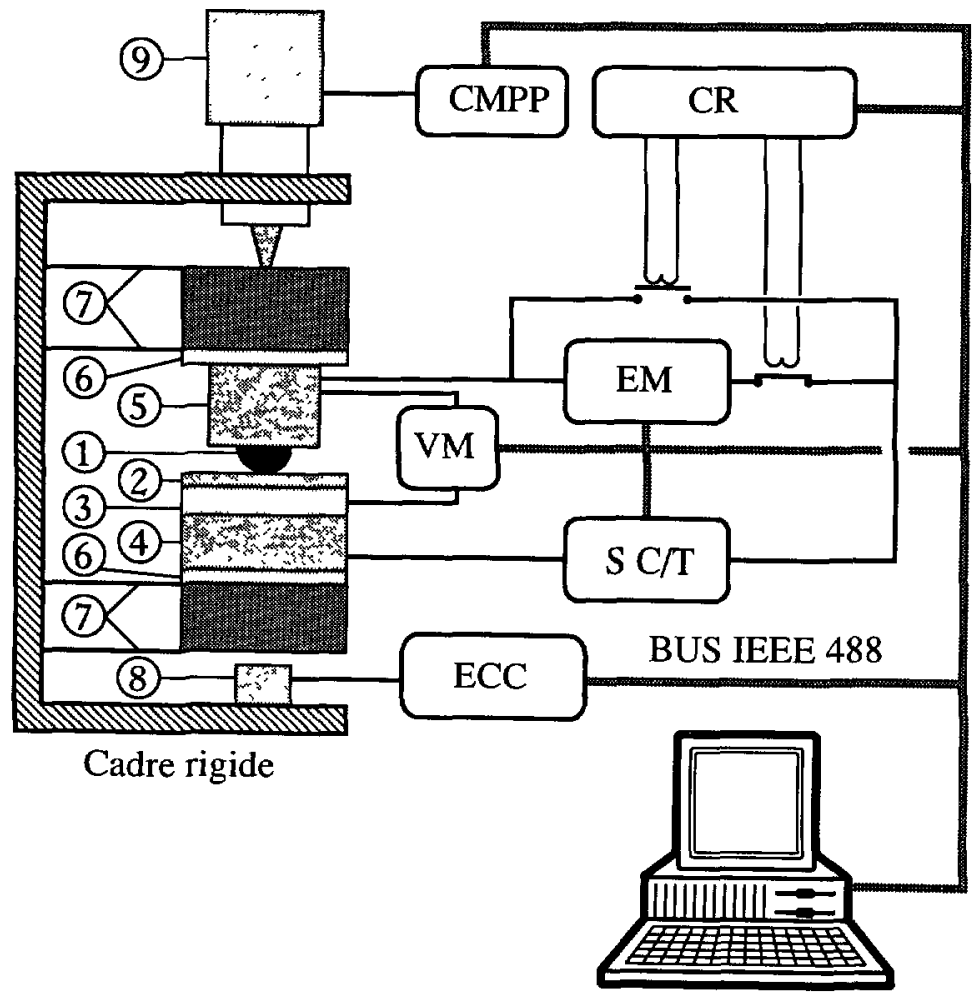

Fig. 7. - Dispositif expérimental utilisé pour les mesures de résistance de contact. (1): Bille dorée polie, (2) : Couche de polymère, (3) : Sous-couche de nickel, (4) : Substrat de laiton, (5) : Porte-bille, (6) : Isolant, (7) : Diaphragmes élastiques, (8) : Capteur capacitif, (9) : Moteur pas-à-pas, CMPP : Commande du moteur pas-à-pas, CR : Commande des relais, EM : Électromètre, VM : Voltmètre, S $\mathrm{C} / \mathrm{T}$ : Source de courant/tension, ECC : Électronique du capteur capacitif.

[Experimental device used for ball/plane contact resistance measurements. (1): Polished golden ball, (2): Polymer layer, (3): Nickel underlayer, (4): Brass substrate, (5): Ball holder, (6): Insulator, (7): Elastic washers, (8): Capacitive sensor, (9): Stepping motor, CMPP: Stepping motor control unit, CR: Relays control unit, EM: Electrometer, VM: Voltmeter, S C/T: Voltage/Current source, ECC: Capacitance measurement unit.] 


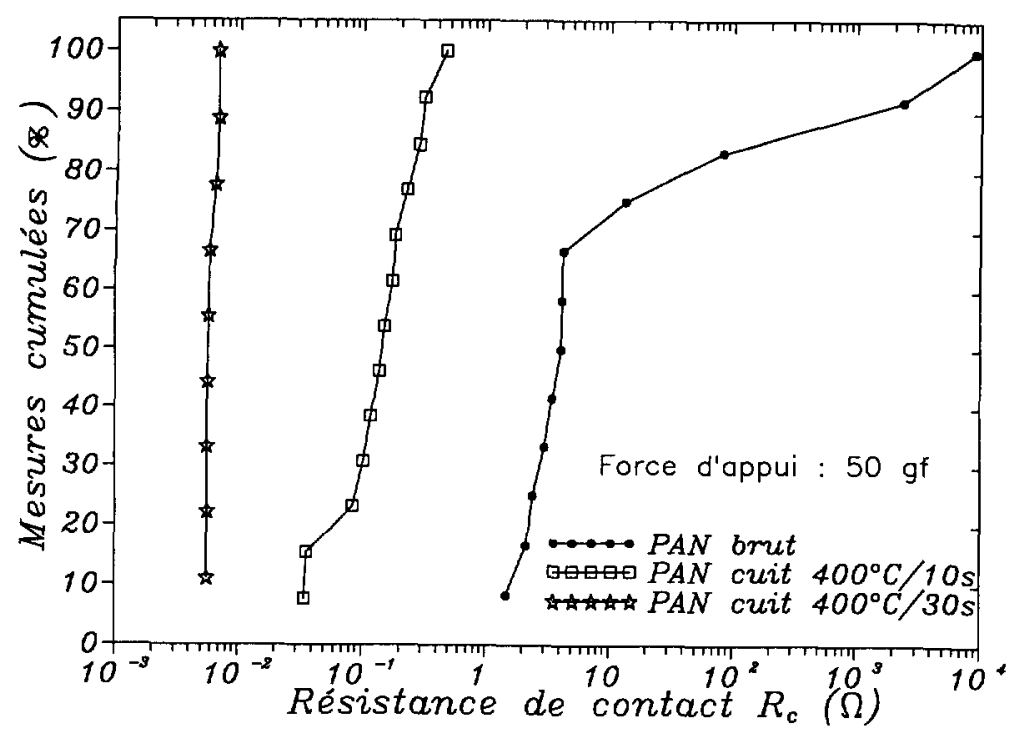

Fig. 8. - Récapitulation des mesures de résistance de contact obtenues avec une force de contact de $50 \mathrm{gf}$ pour trois des échantillons étudiés. (a) : PAN cru ; (b) : PAN cuit $400{ }^{\circ} \mathrm{C} / 10 \mathrm{~s}$; (c) : PAN cuit $400{ }^{\circ} \mathrm{C} / 30 \mathrm{~s}$.

[Summary of the contact resistance results obtained for three studied samples under 50 gf normal load. (a): Raw PAN; (b): PAN heated $400{ }^{\circ} \mathrm{C} / 10 \mathrm{~s}$; (c): PAN heated $400^{\circ} \mathrm{C} / 30 \mathrm{~s}$.]

et à force d'appui constante de 50 gf. Selon le caractère isolant ou conducteur de l'échantillon, on travaille à différence de potentiel imposée ou à courant imposé.

La figure 8 donne le diagramme récapitulatif des mesures réalisées sur trois films de nature différente, un brut et deux cuits. Nous constatons une diminution très nette de la résistance des films résultant de la cuisson. Partant ainsi d'une résistance d'au mieux $1 \Omega$ pour un film cru, on atteint pour l'échantillon cuit à $400^{\circ} \mathrm{C}$ pendant $30 \mathrm{~s}$ des valeurs toutes inférieures à $7 \mathrm{~m} \Omega$. Ces résultats mettent également en évidence la diminution de la dispersion des valeurs mesurées avec la cuisson. Le film cru présente des valeurs de résistance de contact qui s'échelonnent de 1 à $10^{4} \Omega$, tandis que le film le plus cuit $\left(400^{\circ} \mathrm{C} / 30 \mathrm{~s}\right)$ voit toutes ses valeurs comprises entre 5 et $7 \mathrm{~m} \Omega$.

La grande dispersion des valeurs obtenues pour le film cru est probablement due à des hétérogénéités présentes au sein du matériau. La présence de molécules résiduelles de solvant ou d'impuretés, d'irrégularités structurales du polymère (liaisons interchaînes), de variations de densité des chaînes peut créer des barrières pour le transfert électronique et gêner ainsi le passage du courant à travers le polymère. Le recuit du film homogénéiserait le polymère au cours de la modification chimique, conduisant à des résultats beaucoup plus reproductibles.

La forte diminution des résistances de contact observées est tout à fait compatible avec les évolutions de la résistivité du PAN cuit rapportées dans la littérature. Plusieurs effets cumulés peuvent conduire à de telles performances et ont été discutés dans la référence [3]. Tout d'abord, la transformation chimique du polymère crée une structure moléculaire conjuguée où les états électroniques sont délocalisés le long du réseau réticulé. Une telle structure favorise indiscutablement le transport électronique dans le matériau. Ensuite, la diminution importante de l'épaisseur des films (jusqu'à $60 \%$ pour l'échantillon le plus cuit) contribue à faciliter le passage du courant. Enfin, les effets de la pression, qui peut être énorme localement (plusieurs 


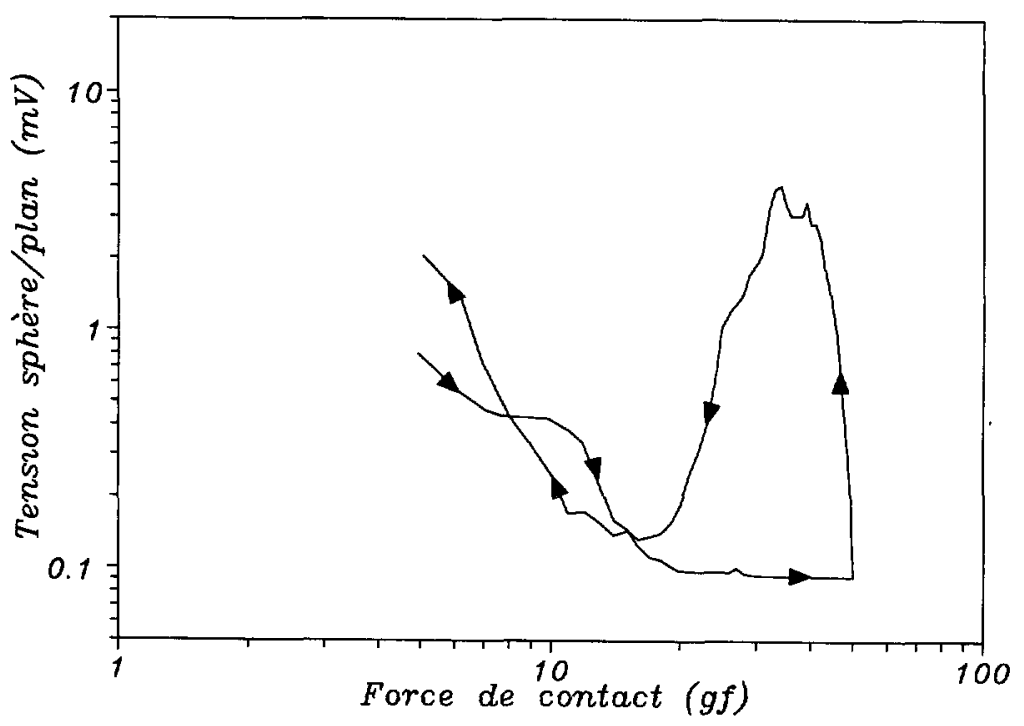

Fig. 9. - Courbe montrant l'évolution typique de la différence de potentiel sphère/plan en fonction de la force de contact pour un échantillon traité à $500^{\circ} \mathrm{C}$ pendant $10 \mathrm{~s}$ (courant imposé de $10 \mathrm{~mA}$ ). [Typical curve showing the contact voltage versus the applied load for a $500{ }^{\circ} \mathrm{C} / 10 \mathrm{~s}$ heat-treated sample with an imposed current of $10 \mathrm{~mA}$.]

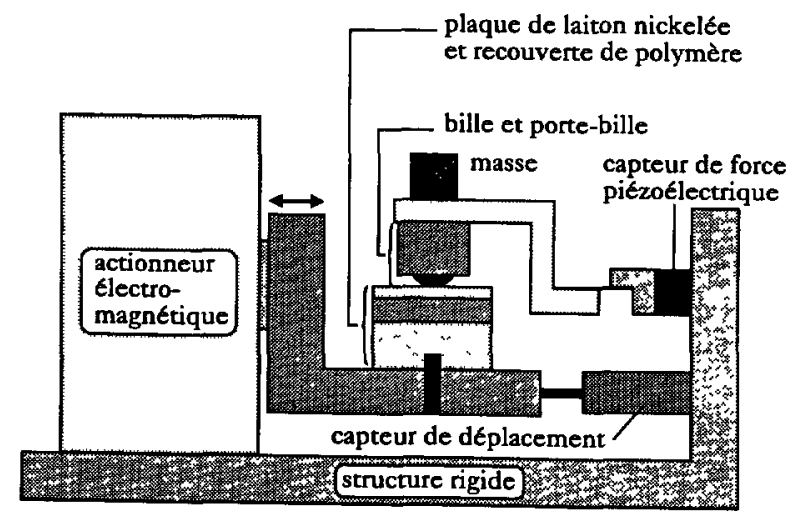

Fig. 10. - Dispositif expérimental utilisé pour les tests tribologiques.

[Experimental set-up used for the tribological tests.]

kbars) en raison de la rugosité des deux éléments du contact, participent probablement aussi aux faibles résistivités obtenues [12].

Afin de vérifier que la bille ne perce pas le film sous la pression exercée, nous avons réalisé des cycles de mesure de la résistance de contact en fonction de la force d'appui sur les films les plus fins (Fig. 9). Lors d'un cycle de chargement/déchargement, une forte hystérésis apparaît, caractéristique du comportement plasto-visco-élastique des polymères. 


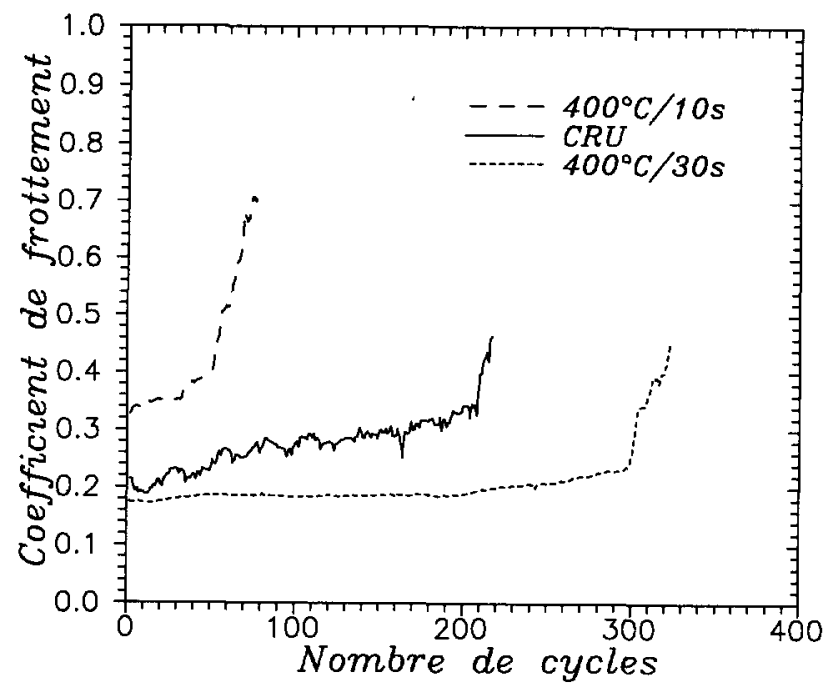

Fig. 11. - Courbe montrant l'évolution du coefficient de frottement moyen en fonction du nombre de cycles réalisés pour trois films de PAN ayant subi différents traitements thermiques. (a) : PAN cru ; (b) : PAN cuit $400{ }^{\circ} \mathrm{C} / 10 \mathrm{~s} ;$ (c) : PAN cuit $400{ }^{\circ} \mathrm{C} / 30 \mathrm{~s}$.

[Plot of the mean friction coefficient versus the number of cycles for three PAN samples submitted to different heat-treatments. (a): Raw PAN; (b): PAN heated $400^{\circ} \mathrm{C} / 10 \mathrm{~s}$; (c): PAN heated $400^{\circ} \mathrm{C} / 30 \mathrm{~s}$.]

Ce résultat lève tout doute sur l'éventuelle existence de jonctions métal/métal.

5.2. PropriÉtÉs tribologiques. - La figure 10 montre le dispositif mis au point au LGEP pour étudier le comportement en frottement des revêtements. Ce test simule les contraintes subies par un contact de connecteur soumis de manière répétée à des insertions/extractions en cours d'utilisation.

Lors des tests tribologiques, une bille effectue sur le plan des aller-retours de $1 \mathrm{~mm}$ d'amplitude avec une vitesse de $0,1 \mathrm{~mm} / \mathrm{s}$, sous une force normale $F_{\mathrm{n}}$ constante de $100 \mathrm{gf}$. Au cours de chaque cycle, on enregistre 2000 valeurs de la force tangentielle $F_{\mathrm{t}}$ qui permettent de calculer, à la fin de chaque cycle, le coefficient de frottement moyen $\mu=\left\langle F_{\mathrm{t}}\right\rangle / F_{\mathrm{n}}$. Des études préliminaires [13] nous ont conduit à réaliser ces tests avec des billes en bronze au béryllium que nous préférons aux billes dorées ou nickelées. Ces dernières ne nous ont en effet pas permis d'obtenir de résultats différenciés : l'or ductile se dépose sur le film sans l'user, tandis que le nickel, qui possède une forte affinité pour le PAN, adhère fortement au film et l'arrache en quelques cycles.

Sur la figure 11, nous voyons l'évolution de $\mu$ en fonction du nombre de cycles pour les trois types de films étudiés au paragraphe 5.1. Le test de frottement se prolonge jusqu'à ce que le coefficient de frottement, faible pour les premiers cycles $(0,1$ à 0,3$)$, atteigne la valeur correspondant au frottement nickel sur cuivre dans nos conditions $(0,5)$ et traduise ainsi la dégradation du film.

Nous constatons que le PAN cru donne un coefficient de frottement initial $\mu_{\mathrm{i}}$ voisin de 0,2 qui augmente ensuite régulièrement. La dégradation complète de ce film intervient vers 200 cycles de frottement.

Le film traité à $400^{\circ} \mathrm{C} / 10 \mathrm{~s}$ montre un $\mu$ initial relativement fort, proche de 0,3 . Le test de frottement aboutit dans ce cas à une rapide dégradation du polymère en 70 cycles environ. Un traitement thermique poussé $\left(400^{\circ} \mathrm{C} / 30 \mathrm{~s}\right)$ améliore le comportement en frottement des 
échantillons en conduisant à la fois à un faible coefficient de frottement initial $\left(\mu_{\mathrm{i}}=0,2\right)$ et à la dégradation retardée du film qui ne survient qu'au bout de 300 cycles.

On voit que le comportement tribologique des films dépend lui aussi beaucoup du temps de traitement thermique. Des éléments d'interprétation de ces résultats peuvent être apportés par les analyses physico-chimiques des échantillons.

Le film cru est constitué de chaînes linéaires de polymère qui, à la différence des structures réticulées, ne sont pas liées par de fortes liaisons chimiques interchaînes. La cohésion des macromolécules est faible et le polymère se dégrade progressivement. La présence d'impuretés hydrocarbonées adsorbées à la surface du film pourrait expliquer la valeur relativement faible de $\mu_{\mathrm{i}}$.

L'échantillon cuit modérément $\left(400^{\circ} \mathrm{C} / 10 \mathrm{~s}\right)$ a une épaisseur plus faible et une surface plus lisse conduisant à une aire de contact supérieure. Cependant sa transformation chimique n'est pas importante : le polymère reste fragile et sa dégradation intervient rapidement. De plus, le film possède encore en surface de nombreux groupements nitriles polaires qui peuvent favoriser une forte adhésion de la bille sur le polymère. En effet, la présence d'azote à la surface d'un polymère augmente le terme polaire de son énergie de surface et peut favoriser son adhésion [14]. L'augmentation de l'adhésion entre la bille et le film pourrait expliquer les valeurs plus élevées de $\mu_{\mathrm{i}}$.

Le traitement thermique poussé $\left(400^{\circ} \mathrm{C} / 30 \mathrm{~s}\right)$ conduit à former une structure réticulée dense et compacte qui améliore la solidité du film. Celui-ci résiste donc mieux aux contraintes exercées par la bille au cours du frottement. De plus, la surface des films s'est largement appauvrie en azote, comme le montrent les analyses XPS indiquant une diminution de $20 \%$ de la quantité d'azote présent en surface du polymère.

\section{Conclusion}

Par un procédé de traitement thermique rapide, nous avons obtenu, à partir de PAN électropolymérisé, une couche mince de polymère dont les propriétés électriques et tribologiques sont très prometteuses pour diverses applications comme la connectique, ou la lubrification en milieu extrême (vide...).

Les investigations menées au niveau moléculaire, microscopique et macroscopique nous ont permis de corréler la composition chimique et la structure des films avec leur aspect topographique et leurs intéressantes performances électriques et tribologiques. L'approche multidisciplinaire utilisée montre que, dans la mécanique d'un contact faisant intervenir un polymère, de nombreux phénomènes complexes interviennent et influent sur son comportement électrique et tribologique. Les recherches à venir auront pour but de préciser les mécanismes de transport électronique dans les films, ainsi que les phénomènes de plasto-visco-élasticité qui peuvent avoir lieu sous l'effet d'une contrainte mécanique.

\section{Bibliographie}

[1] Lécayon G., Viel P., Le Gressus C., Boiziau C., Leroy S., Perreau J. et Reynaud C., A metalpolymer interface study using electropolymerized acrylonitrile on nickel surfaces, Scan. Microsc. 1 (1987) 85.

[2] Lécayon G., Viel P., Boissel J. et Delhalle J., Contact for electrical connector coated with a polymer film and its fabrication process, Brevet $n^{\circ} 9101604(12 / 02 / 1991)$. 
[3] Houzé F., Boyer L., Noël S., Viel P., Lécayon G. et Bourin J-M., Electrical properties of very thin heat-treated polyacrylonitrile layers electropolymerized on nickel for contact application, Synth. Metals 62 (1994) 207.

[4] Fitzer E., Pan-based carbon fibers - present state and trend of the technology from the viewpoint of possibilities and limits to influence and to control the fiber properties by the process parameters, Carbon 27 (1989) 621.

[5] Teoh H., Metz P.D. et Wilhelm W.G., Electrical conductivity of pyrolysed polyacrylonitrile, Mol. Cryst. Liq. Cryst. 83 (1982) 297.

[6] Shimada I. et Takahagi T., FT-IR study of the stabilization reaction of polyacrylonitrile in the production of carbon fibres, J. Polym. Scr. Polym. Chem. Ed. 24 (1986) 1989.

[7] Clarke A.J. et Bailey J.E., Oxidation of acrylic fibres for carbon fibre formation, Nat. 24 (1973) 146.

[8] Mathieu D., Defranceschi M., Viel P., Lécayon G. et Delhalle J., Theoretical and experimental study of the molecular structure absorbing at $2190 \mathrm{~cm}^{-1}$ in electroinitiated polyacrylonitrile, Proc. of the International Conference on Polymer-Solid Interface (IOP Publishing Ltd, 1992) p. 379.

[9] Leroy S., Boiziau C., Perreau J., Reynaud C., Zalczer G., Lécayon G. et Le Gressus C., Molecular structure of an electropolymerized PAN film and its pyrolysed derivatives, J. Mol. Struct. 128 (1985) 269.

[10] Krigbaum W.R. et Tokita N., Melting point depression study of polyacrylonitrile, J. Polym. Scz. 43 (1960) 467.

[11] Dunn P. et Ennis B.C., Thermal analysis of polyacrylonitrile : the melting point of polyacrylonitrile, J. Appl. Polym. Scr. 14 (1970) 1795.

[12] Isotalo H., Ahlskog M. et Stubb H., Pressure dependence of d.c. conductivity in poly(3alkylthiophenes), Synth. Metals 48 (1992) 313.

[13] Noël S., Newton P., Houzé F., Boyer L., Viel P. et Lécayon G., Propriétés mécaniques de films de polyacrylonitrile électropolymérisés soumis à des essais tribologiques : influence d'un recuit rapide, Le vide, les couches minces 268 (suppl.) (1993) 143.

[14] Datta P. et Kaganowicz G., Adhesion of thin plasma-polymerized films to metals, Polym. sci. technol., 12B (LEE, Ed., Plenum Press, Londres, 1980). 